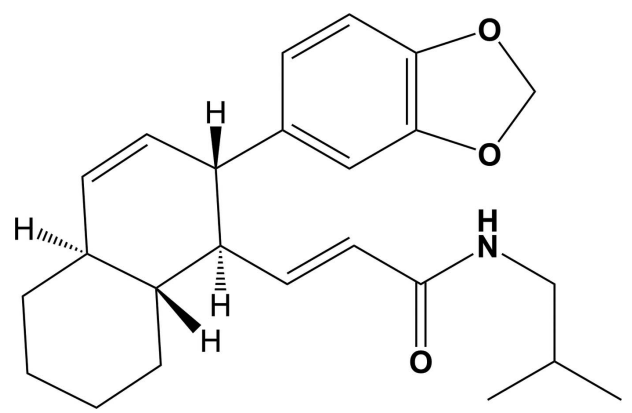

\title{
Crystal structure of obscurine: a natural product isolated from the stem bark of
}

\section{B. obscura}

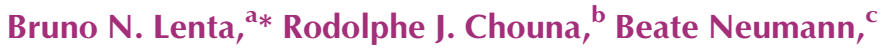 \\ Hans-Georg Stammler ${ }^{c}$ and Norbert Sewald ${ }^{c}$ \\ a Department of Chemistry, Higher Teacher Training College, University of Yaoundé \\ 1, PO Box 47, Yaoundé, Cameroon, ${ }^{\mathbf{b}}$ Department of Chemistry, University of \\ Dschang, PO Box 371, Dschang, Cameroon, and ${ }^{\mathrm{C}}$ Department of Chemistry, \\ University of Bielefeld, PO Box 100131, 33501 Bielefeld, Germany. *Correspon- \\ dence e-mail: lentabruno@yahoo.fr
}

Received 21 April 2015; accepted 1 June 2015

Edited by H. Stoeckli-Evans, University of Neuchâtel, Switzerland

The title compound, $\mathrm{C}_{24} \mathrm{H}_{31} \mathrm{NO}_{3}$ \{systematic name: $(E)-3$ $\left[\left(1 R^{*}, 2 S^{*}, 4 \mathrm{a} S^{*}, 8 \mathrm{a} R^{*}\right)-2-(\right.$ benzo[ $[d][1,3]$ dioxol-5-yl)-1,2,4a,5,6,7,8,8 a-octahydronaphthalen-1-yl]- $N$-isobutylacrylamide\}, is a natural product isolated from the stem bark of B. obscura. It is composed of an octahydronaphthalene ring system substituted with an essentially planar benzodioxole ring system [r.m.s. deviation $=0.012 \AA$ ] and an extended isobutylacrylamide group. In the crystal, molecules are linked by $\mathrm{N}-$ $\mathrm{H}$... O hydrogen bonds, forming chains propagating along [100]. The chains are linked by pairs of $\mathrm{C}-\mathrm{H} \cdots \mathrm{O}$ hydrogen bonds, involving inversion-related benzodioxole ring systems, forming ribbons lying parallel to (010). There are also $\mathrm{C}-$ $\mathrm{H} \cdots \pi$ interactions present within the ribbons.

Keywords: crystal structure; obscurine; octahydronaphthalene; benzodioxole; isobutylacrylamide; $\mathrm{N}-\mathrm{H} \cdots \mathrm{O}$ hydrogen bonds.

CCDC reference: 1404418

\section{Related literature}

For background to the Beilschmiedia genus, medicinal plants used in Cameroon, see: Chouna et al. (2009, 2010, 2011); Lenta et al. (2009, 2011). For related structures, see: Balawsnt et al. (1975).

\section{Experimental}

2.1. Crystal data

$\mathrm{C}_{24} \mathrm{H}_{31} \mathrm{NO}_{3}$

$M_{r}=381.50$

Triclinic, $P \overline{1}$

$a=5.14153(16) \AA$

$b=9.7449(3) \AA$

$c=20.4639(5) \AA$

$\alpha=98.839$ (2)

$\beta=90.946(2)^{\circ}$

\subsection{Data collection}

Agilent SuperNova, Dual, $\mathrm{Cu}$ at zero, Atlas diffractometer

Absorption correction: gaussian (CrysAlis PRO; Agilent, 2013)

$T_{\min }=0.798, T_{\max }=1.000$

\subsection{Refinement}

$R\left[F^{2}>2 \sigma\left(F^{2}\right)\right]=0.035$

$w R\left(F^{2}\right)=0.091$

$S=1.04$

3892 reflections

Table 1

Hydrogen-bond geometry $\left(\AA{ }^{\circ}\right)$.

$\mathrm{Cg} 2$ is the centroid of the benzene ring $\mathrm{C} 2-\mathrm{C} 7$.

\begin{tabular}{lllll}
\hline$D-\mathrm{H} \cdots A$ & $D-\mathrm{H}$ & $\mathrm{H} \cdots A$ & $D \cdots A$ & $D-\mathrm{H} \cdots A$ \\
\hline $\mathrm{N} 1-\mathrm{H} 1 \cdots \mathrm{O} 3^{\mathrm{i}}$ & $0.896(16)$ & $2.105(16)$ & $2.8938(13)$ & $146.3(13)$ \\
$\mathrm{C} 7-\mathrm{H} 7 \cdots \mathrm{O} 1^{\mathrm{ii}}$ & $0.984(16)$ & $2.503(16)$ & $3.4264(15)$ & $156.3(12)$ \\
$\mathrm{C} 1-\mathrm{H} 1 B \cdots C g 2^{\mathrm{i}}$ & $0.978(16)$ & $2.595(15)$ & $3.4578(12)$ & $147.4(11)$ \\
\hline Symmetry codes: (i) $x-1, y, z ;(\mathrm{ii})-x+1,-y+2,-z+1$. &
\end{tabular}

Data collection: CrysAlis PRO (Agilent, 2013); cell refinement: CrysAlis PRO; data reduction: CrysAlis PRO; program(s) used to solve structure: SHELXS97 (Sheldrick, 2008); program(s) used to refine structure: SHELXL97 (Sheldrick, 2008); molecular graphics: Mercury (Macrae et al., 2008); software used to prepare material for publication: OLEX2 (Dolomanov et al., 2009) and PLATON (Spek, 2009).

\section{Acknowledgements}

BNL thanks the Alexander von Humboldt Foundation for providing a fellowship to study at Bielefeld University. 
Supporting information for this paper is available from the $\mathrm{IUCr}$ electronic archives (Reference: SU5126).

\section{References}

Agilent (2013). CrysAlis PRO. Agilent Technologies, Yarnton, England.

Balawsnt, S. J., Nerayanan, V., Dillip, H. G., Veakstachahm, B. \& Wolfgang, V. P. (1975). Helv. Chim. Acta, 58, 2295-2305.

Chouna, J. R., Nkeng-Efouet, P. A., Lenta, B. N., Wansi, D. J., Neumann, B., Stammler, H.-G., Fon Kimbu, S. \& Sewald, N. (2011). Helv. Chim. Acta, 94, 1071-1076.

Chouna, J. R., Nkeng-Efouet, P. A., Lenta, B. N., Devkota, K. P., Neumann, B., Stammler, H. G., Kimbu, S. F. \& Sewald, N. (2009). Phytochemistry, 70, 684688.
Chouna, J. R., Nkeng-Efouet, P. A., Lenta, B. N., Wansi, J. D., Kimbu, S. F. \& Sewald, N. (2010). Phytochemistry Lett. 3, 13-16.

Dolomanov, O. V., Bourhis, L. J., Gildea, R. J., Howard, J. A. K. \& Puschmann, H. (2009). J. Appl. Cryst. 42, 339-341.

Lenta, B. N., Chouna, J. R., Nkeng-Efouet, P. A., Kimbu, S. F., Tsamo, E. \& Sewald, N. (2011). Nat. Prod. Commun. 6, 1591-1592.

Lenta, B. N., Tantangmo, F., Devkota, K. P., Wansi, J. D., Chouna, J. R., Soh, R. C. F., Neumann, B., Stammler, H.-G., Tsamo, E. \& Sewald, N. (2009). J. Nat. Prod. 72, 2130-2134.

Macrae, C. F., Bruno, I. J., Chisholm, J. A., Edgington, P. R., McCabe, P., Pidcock, E., Rodriguez-Monge, L., Taylor, R., van de Streek, J. \& Wood, P. A. (2008). J. Appl. Cryst. 41, 466-470.

Sheldrick, G. M. (2008). Acta Cryst. A64, 112-122.

Spek, A. L. (2009). Acta Cryst. D65, 148-155. 


\section{supporting information}

Acta Cryst. (2015). E71, o457-o458 [doi:10.1107/S2056989015010567]

\section{Crystal structure of obscurine: a natural product isolated from the stem bark of B. obscura}

\section{Bruno N. Lenta, Rodolphe J. Chouna, Beate Neumann, Hans-Georg Stammler and Norbert Sewald}

\section{S1. Chemical context}

Plants of the Beilschmiedia genus have been the subject of research interest (Lenta et al., 2009, 2011; Chouna et al. 2011). Our interests involve the isolation of active constituents from the stem bark and leaves of $B$. obscura, a medicinal plant used in Cameroon. Herein, we report on the crystal structure of the title compound, a natural product isolated from the stem bark of $B$. obscura.

\section{S2. Isolation and crystallization}

The air-dried stem bark of $B$. obscura ( $400 \mathrm{~g}$ ) was macerated with methanol at room temperature for $72 \mathrm{~h}$. The methanol extract was concentrated under reduced pressure to give a residue of $16 \mathrm{~g}$, which was selectively extracted with $\mathrm{CH}_{2} \mathrm{Cl}_{2}$ at room temperature to afford $4 \mathrm{~g}$ of the $\mathrm{CH}_{2} \mathrm{Cl}_{2}$ soluble residue. This extract was subjected to column chromatography (CC) over silica gel (0.023-0.20 mesh, Merck) and eluted with a gradient system of n-hexane/ $\mathrm{CH}_{2} \mathrm{Cl}_{2}$ and $\left(\mathrm{CH}_{2} \mathrm{Cl}_{2} / \mathrm{MeOH}\right.$,) to afford obscurine $(4.2 \mathrm{mg})$, the solid obtained was grounded and then recrystallised from a mixture of petroleum ether/dichlorometahne (1:1), yielding colourless needle-like crystals.

\section{S3. Refinement}

Crystal data, data collection and structure refinement details are summarized in Table 2. All of the $\mathrm{H}$ atoms were all located in difference Fourier maps and freely refined. 


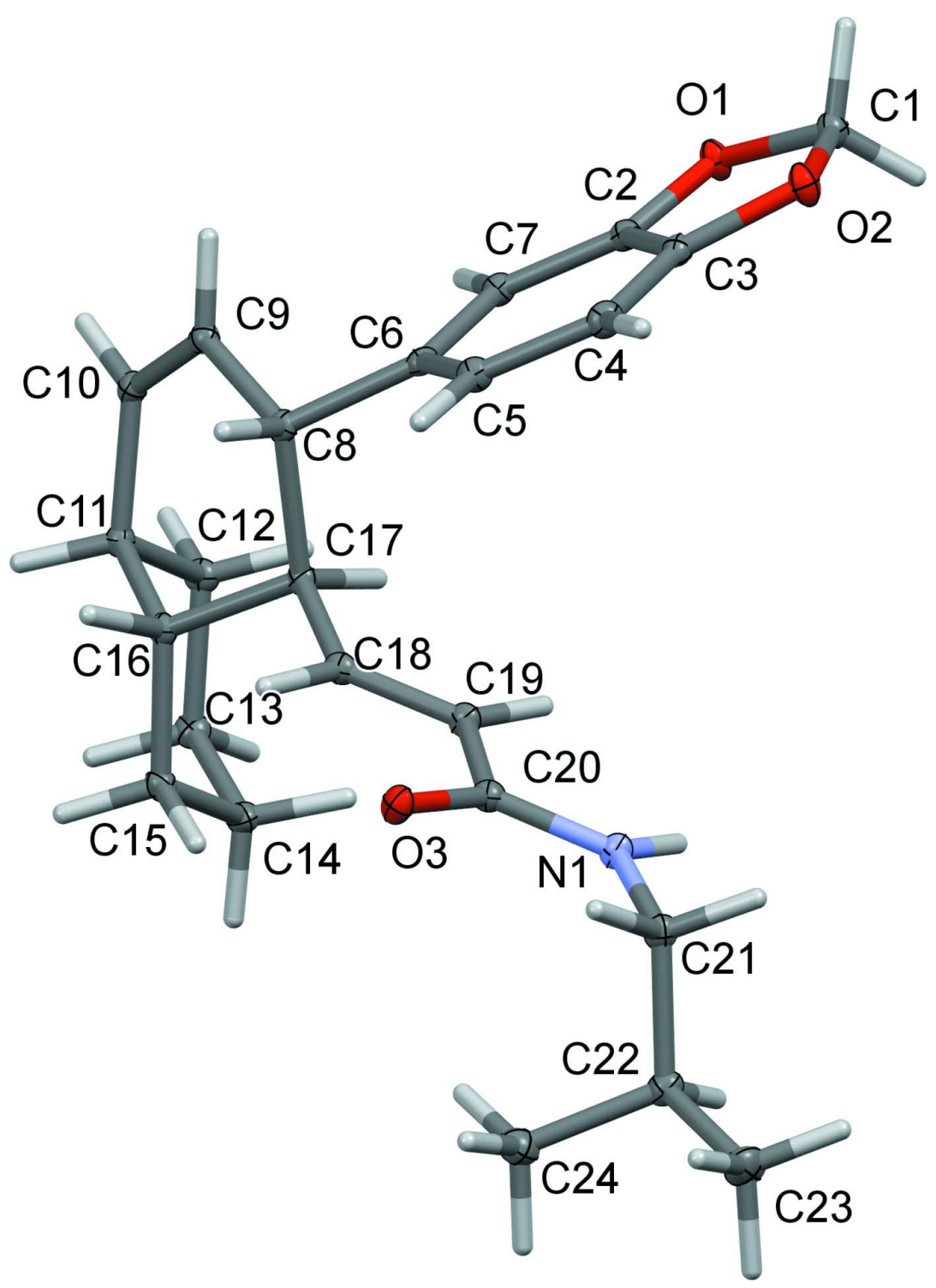

Figure 1

A view of the molecular structure of the title compound, with atom labelling. Displacement ellipsoids are drawn at the $50 \%$ probabilityl evel. 


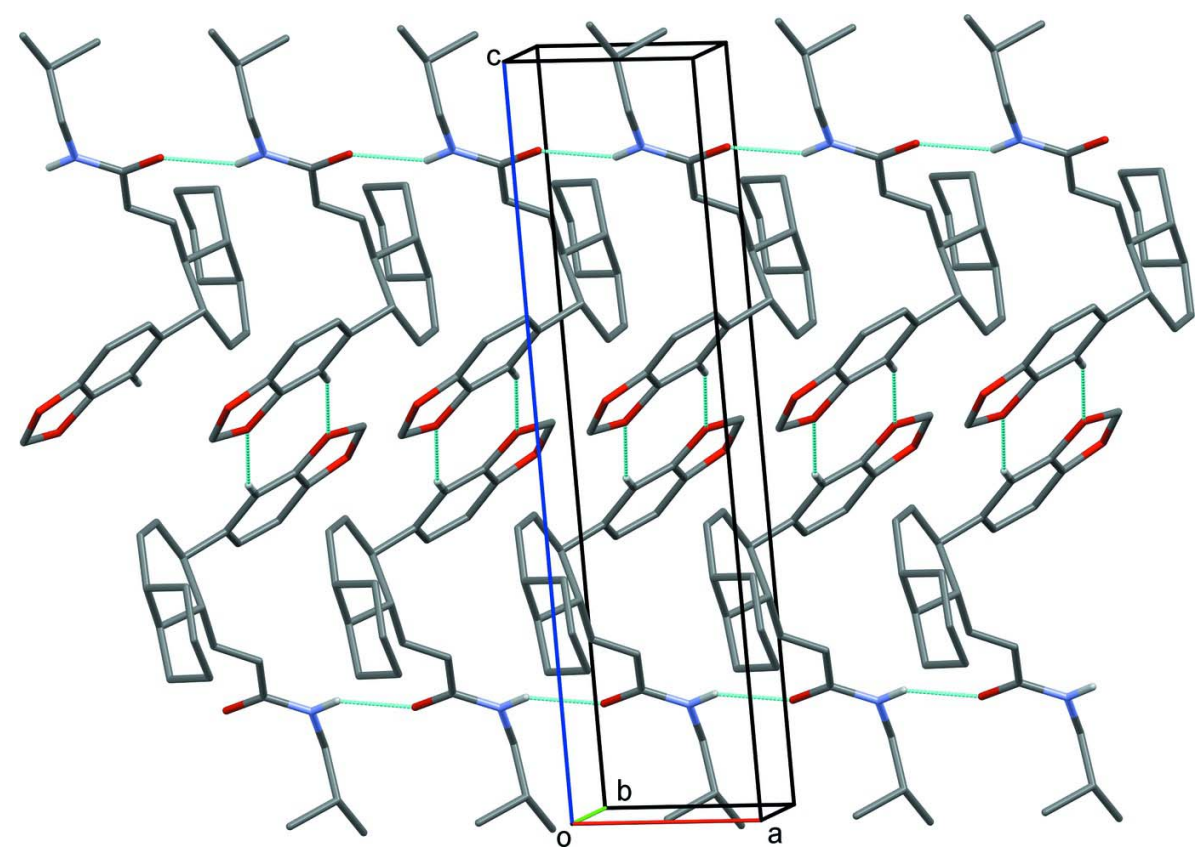

\section{Figure 2}

A view along the $b$ axis of the crystal packing of the title compound. The hydrogen bonds are shown as dashed lines (see Table 1 for details).

\section{(E)-3-[(1 $\left.R^{*}, 2 S^{*}, 4 \mathrm{a} S^{*}, 8 \mathrm{a} R^{*}\right)-2-(B e n z o[d][1,3]$ dioxol-5-yl)-1,2,4a,5,6,7,8,8a-octahydronaphthalen-1-yl]-N-} isobutylacrylamide

Crystal data

$\mathrm{C}_{24} \mathrm{H}_{31} \mathrm{NO}_{3}$

$M_{r}=381.50$

Triclinic, $P \overline{1}$

$a=5.14153(16) \AA$

$b=9.7449(3) \AA$

$c=20.4639(5) \AA$

$\alpha=98.839(2)^{\circ}$

$\beta=90.946(2)^{\circ}$

$\gamma=100.237(3)^{\circ}$

$V=996.00(5) \AA^{3}$

\section{Data collection}

Agilent SuperNova, Dual, Cu at zero, Atlas diffractometer

Radiation source: SuperNova $(\mathrm{Cu}) \mathrm{X}$-ray Source

Mirror monochromator

Detector resolution: 5.3114 pixels $\mathrm{mm}^{-1}$

$\omega$ scans

Absorption correction: gaussian

(CrysAlis PRO; Agilent, 2013)
$Z=2$

$F(000)=412$

$D_{\mathrm{x}}=1.272 \mathrm{Mg} \mathrm{m}^{-3}$

$\mathrm{Cu} K \alpha$ radiation, $\lambda=1.5418 \AA$

Cell parameters from 7209 reflections

$\theta=4.7-76.2^{\circ}$

$\mu=0.66 \mathrm{~mm}^{-1}$

$T=100 \mathrm{~K}$

Needle, clear colourless

$0.36 \times 0.03 \times 0.02 \mathrm{~mm}$

$T_{\min }=0.798, T_{\max }=1.000$

13324 measured reflections

3892 independent reflections

3425 reflections with $I>2 \sigma(I)$

$R_{\text {int }}=0.027$

$\theta_{\max }=72.1^{\circ}, \theta_{\min }=4.4^{\circ}$

$h=-6 \rightarrow 5$

$k=-11 \rightarrow 12$

$l=-24 \rightarrow 24$ 


\section{Refinement}

Refinement on $F^{2}$

Least-squares matrix: full

$R\left[F^{2}>2 \sigma\left(F^{2}\right)\right]=0.035$

$w R\left(F^{2}\right)=0.091$

$S=1.04$

3892 reflections

377 parameters

0 restraints
Primary atom site location: structure-invariant direct methods

Hydrogen site location: difference Fourier map

All H-atom parameters refined

$w=1 /\left[\sigma^{2}\left(F_{\mathrm{o}}^{2}\right)+(0.0424 P)^{2}+0.3477 P\right]$

where $P=\left(F_{\mathrm{o}}^{2}+2 F_{\mathrm{c}}^{2}\right) / 3$

$(\Delta / \sigma)_{\max }=0.001$

$\Delta \rho_{\max }=0.24 \mathrm{e} \AA^{-3}$

$\Delta \rho_{\min }=-0.22$ e $\AA^{-3}$

Special details

Geometry. All e.s.d.'s (except the e.s.d. in the dihedral angle between two 1.s. planes) are estimated using the full covariance matrix. The cell e.s.d.'s are taken into account individually in the estimation of e.s.d.'s in distances, angles and torsion angles; correlations between e.s.d.'s in cell parameters are only used when they are defined by crystal symmetry. An approximate (isotropic) treatment of cell e.s.d.'s is used for estimating e.s.d.'s involving 1.s. planes.

Fractional atomic coordinates and isotropic or equivalent isotropic displacement parameters $\left(\AA^{2}\right)$

\begin{tabular}{|c|c|c|c|c|}
\hline & $x$ & $y$ & $z$ & $U_{\text {iso }} * / U_{\text {eq }}$ \\
\hline $\mathrm{O} 3$ & $0.99052(16)$ & $0.81354(9)$ & $0.86676(4)$ & 0.01947 (19) \\
\hline O1 & $0.32116(17)$ & $0.81350(9)$ & $0.50886(4)$ & $0.0198(2)$ \\
\hline $\mathrm{O} 2$ & $0.26950(17)$ & $0.59230(9)$ & $0.54145(4)$ & $0.0211(2)$ \\
\hline N1 & $0.5440(2)$ & $0.79089(11)$ & $0.87047(5)$ & $0.0177(2)$ \\
\hline $\mathrm{C} 7$ & $0.7117(2)$ & $0.92262(12)$ & $0.58231(6)$ & $0.0155(2)$ \\
\hline C6 & $0.8900(2)$ & $0.89637(12)$ & $0.62993(6)$ & $0.0149(2)$ \\
\hline $\mathrm{C} 14$ & $0.9558(2)$ & $1.40385(13)$ & $0.81143(6)$ & 0.0189 \\
\hline $\mathrm{C} 18$ & $0.9906(2)$ & $1.00932(12)$ & $0.77808(6)$ & $0.0161(2)$ \\
\hline C19 & $0.7706(2)$ & $0.94294(12)$ & $0.80006(6)$ & $0.0172(2)$ \\
\hline $\mathrm{C} 4$ & $0.6528(2)$ & $0.65272(13)$ & $0.62212(6)$ & $0.0183(2)$ \\
\hline $\mathrm{C} 16$ & $1.2263(2)$ & $1.23586(12)$ & $0.74705(6)$ & $0.0152(2)$ \\
\hline $\mathrm{C} 17$ & $1.0186(2)$ & $1.10091(12)$ & $0.72506(6)$ & $0.0147(2)$ \\
\hline $\mathrm{C} 2$ & $0.5136(2)$ & $0.81316(12)$ & $0.55642(6)$ & $0.0154(2)$ \\
\hline $\mathrm{C} 22$ & $0.4536(2)$ & $0.77083(13)$ & $0.98764(6)$ & 0.0195 (3) \\
\hline $\mathrm{C} 8$ & $1.1097(2)$ & $1.01511(12)$ & $0.66128(6)$ & $0.0150(2)$ \\
\hline C3 & $0.4823(2)$ & $0.68151(12)$ & $0.57583(6)$ & $0.0162(2)$ \\
\hline $\mathrm{C} 5$ & $0.8593(2)$ & $0.76299(12)$ & $0.64871(6)$ & $0.0174(2)$ \\
\hline $\mathrm{C} 20$ & $0.7803(2)$ & $0.84434(12)$ & $0.84886(6)$ & $0.0161(2)$ \\
\hline $\mathrm{C} 21$ & $0.5080(2)$ & $0.69546(13)$ & 0.91942 (6) & $0.0182(3)$ \\
\hline $\mathrm{C} 12$ & $1.0346(2)$ & $1.41782(12)$ & $0.69190(6)$ & $0.0174(2)$ \\
\hline C13 & $1.0029(3)$ & $1.50233(13)$ & $0.75965(6)$ & $0.0192(3)$ \\
\hline C9 & $1.2170(2)$ & $1.11024(13)$ & $0.61238(6)$ & $0.0163(2)$ \\
\hline C11 & $1.2589(2)$ & $1.33271(12)$ & $0.69369(6)$ & $0.0153(2)$ \\
\hline C10 & $1.2835(2)$ & $1.24957(13)$ & $0.62661(6)$ & $0.0166(2)$ \\
\hline $\mathrm{C} 23$ & 0.3498 & 0.66495 (16) & $1.03273(7)$ & 0.0293 \\
\hline C15 & $1.1801(2)$ & $1.32087(13)$ & $0.81431(6)$ & $0.0178(2)$ \\
\hline $\mathrm{C} 1$ & $0.1586(2)$ & $0.67567(13)$ & $0.49990(6)$ & $0.0186(3)$ \\
\hline $\mathrm{C} 24$ & $0.6991(3)$ & $0.87158(15)$ & $1.01892(7)$ & 0.0260 \\
\hline H16 & $1.402(3)$ & $1.2038(15)$ & $0.7515(7)$ & $0.016(3)^{*}$ \\
\hline H11 & $1.426(3)$ & $1.4037(15)$ & $0.7059(7)$ & $0.015(3)^{*}$ \\
\hline
\end{tabular}




$\begin{array}{lllll}\text { H17 } & 0.844(3) & 1.1256(15) & 0.7138(7) & 0.015(3)^{*} \\ \text { H21A } & 0.670(3) & 0.6545(15) & 0.9203(7) & 0.019(4)^{*} \\ \text { H15A } & 1.345(3) & 1.3876(15) & 0.8301(7) & 0.017(3)^{*} \\ \text { H4 } & 0.633(3) & 0.5592(17) & 0.6353(8) & 0.026(4)^{*} \\ \text { H9 } & 1.238(3) & 1.0624(15) & 0.5679(7) & 0.018(3)^{*} \\ \text { H5 } & 0.986(3) & 0.7444(16) & 0.6806(8) & 0.023(4)^{*} \\ \text { H12A } & 0.862(3) & 1.3498(16) & 0.6770(7) & 0.019(4)^{*} \\ \text { H18 } & 1.159(3) & 0.9934(15) & 0.7974(7) & 0.020(4)^{*} \\ \text { H7 } & 0.730(3) & 1.0156(17) & 0.5680(7) & 0.022(4)^{*} \\ \text { H13A } & 1.169(3) & 1.5731(16) & 0.7739(8) & 0.023(4)^{*} \\ \text { H14A } & 0.942(3) & 1.4596(16) & 0.8555(8) & 0.023(4)^{*} \\ \text { H21B } & 0.354(3) & 0.6183(16) & 0.9026(7) & 0.022(4)^{*} \\ \text { H1 } & 0.399(3) & 0.8194(16) & 0.8567(7) & 0.022(4)^{*} \\ \text { H1A } & 0.160(3) & 0.6301(16) & 0.4528(8) & 0.023(4)^{*} \\ \text { H8 } & 1.260(3) & 0.9720(15) & 0.6758(7) & 0.019(4)^{*} \\ \text { H12B } & 1.068(3) & 1.4823(16) & 0.6573(8) & 0.021(4)^{*} \\ \text { H14B } & 0.780(3) & 1.3357(16) & 0.7997(8) & 0.023(4)^{*} \\ \text { H1B } & -0.020(3) & 0.6828(15) & 0.5137(7) & 0.021(4)^{*} \\ \text { H22 } & 0.313(3) & 0.8255(15) & 0.9805(7) & 0.018(3)^{*} \\ \text { H10 } & 1.350(3) & 1.3042(16) & 0.5915(8) & 0.023(4)^{*} \\ \text { H15B } & 1.146(3) & 1.2548(17) & 0.8489(8) & 0.025(4)^{*} \\ \text { H13B } & 0.851(3) & 1.5540(17) & 0.7578(8) & 0.026(4)^{*} \\ \text { H24A } & 0.841(3) & 0.8171(19) & 1.0287(9) & 0.036(5)^{*} \\ \text { H24B } & 0.660(3) & 0.9237(19) & 1.0614(9) & 0.037(5)^{*} \\ \text { H23A } & 0.483(4) & 0.609(2) & 1.0412(9) & 0.041(5)^{*} \\ \text { H19 } & 0.599(3) & 0.9535(17) & 0.7834(8) & 0.029(4)^{*} \\ \text { H24C } & 0.775(3) & 0.9404(19) & 0.9890(9) & 0.036(5)^{*} \\ \text { H23B } & 0.304(4) & 0.717(2) & 1.0772(10) & 0.043(5)^{*} \\ \text { H23C } & 0.187(4) & 0.601(2) & 1.0128(9) & 0.043(5)^{*}\end{array}$

Atomic displacement parameters $\left(\AA^{2}\right)$

\begin{tabular}{lllllll}
\hline & $U^{11}$ & $U^{22}$ & $U^{33}$ & $U^{12}$ & $U^{13}$ & $U^{23}$ \\
\hline O3 & $0.0158(4)$ & $0.0234(4)$ & $0.0221(4)$ & $0.0068(3)$ & $0.0012(3)$ & $0.0090(3)$ \\
O1 & $0.0191(4)$ & $0.0170(4)$ & $0.0230(4)$ & $0.0005(3)$ & $-0.0059(3)$ & $0.0060(3)$ \\
O2 & $0.0198(4)$ & $0.0153(4)$ & $0.0277(5)$ & $-0.0001(3)$ & $-0.0058(4)$ & $0.0058(3)$ \\
N1 & $0.0154(5)$ & $0.0214(5)$ & $0.0187(5)$ & $0.0052(4)$ & $0.0010(4)$ & $0.0087(4)$ \\
C7 & $0.0162(6)$ & $0.0148(5)$ & $0.0173(6)$ & $0.0050(4)$ & $0.0025(4)$ & $0.0048(4)$ \\
C6 & $0.0153(5)$ & $0.0152(5)$ & $0.0152(5)$ & $0.0049(4)$ & $0.0034(4)$ & $0.0031(4)$ \\
C14 & $0.0176(6)$ & $0.0216(6)$ & $0.0169(6)$ & $0.0042(5)$ & $0.0017(5)$ & $0.0007(5)$ \\
C18 & $0.0175(6)$ & $0.0160(5)$ & $0.0157(6)$ & $0.0054(4)$ & $-0.0008(4)$ & $0.0029(4)$ \\
C19 & $0.0161(6)$ & $0.0199(6)$ & $0.0176(6)$ & $0.0060(5)$ & $0.0000(4)$ & $0.0059(4)$ \\
C4 & $0.0207(6)$ & $0.0143(6)$ & $0.0218(6)$ & $0.0047(5)$ & $0.0027(5)$ & $0.0069(4)$ \\
C16 & $0.0134(5)$ & $0.0160(5)$ & $0.0171(6)$ & $0.0040(4)$ & $-0.0006(4)$ & $0.0038(4)$ \\
C17 & $0.0134(5)$ & $0.0166(6)$ & $0.0154(6)$ & $0.0048(4)$ & $-0.0001(4)$ & $0.0041(4)$ \\
C2 & $0.0153(5)$ & $0.0175(6)$ & $0.0150(5)$ & $0.0059(4)$ & $0.0018(4)$ & $0.0043(4)$ \\
C22 & $0.0195(6)$ & $0.0213(6)$ & $0.0200(6)$ & $0.0066(5)$ & $0.0031(5)$ & $0.0070(5)$ \\
C8 & $0.0138(5)$ & $0.0154(5)$ & $0.0170(6)$ & $0.0049(4)$ & $0.0006(4)$ & $0.0038(4)$
\end{tabular}




\begin{tabular}{lllllll} 
C3 & $0.0147(5)$ & $0.0146(5)$ & $0.0188(6)$ & $0.0020(4)$ & $0.0022(4)$ & $0.0019(4)$ \\
C5 & $0.0187(6)$ & $0.0172(6)$ & $0.0185(6)$ & $0.0067(5)$ & $0.0006(5)$ & $0.0056(4)$ \\
C20 & $0.0175(6)$ & $0.0158(5)$ & $0.0156(6)$ & $0.0043(4)$ & $0.0004(4)$ & $0.0027(4)$ \\
C21 & $0.0194(6)$ & $0.0169(6)$ & $0.0193(6)$ & $0.0027(5)$ & $0.0008(5)$ & $0.0068(4)$ \\
C12 & $0.0185(6)$ & $0.0172(6)$ & $0.0181(6)$ & $0.0060(5)$ & $-0.0004(5)$ & $0.0045(4)$ \\
C13 & $0.0198(6)$ & $0.0172(6)$ & $0.0218(6)$ & $0.0070(5)$ & $0.0003(5)$ & $0.0022(5)$ \\
C9 & $0.0141(6)$ & $0.0189(6)$ & $0.0164(6)$ & $0.0039(4)$ & $0.0017(4)$ & $0.0032(4)$ \\
C11 & $0.0135(5)$ & $0.0154(5)$ & $0.0171(6)$ & $0.0025(4)$ & $0.0006(4)$ & $0.0033(4)$ \\
C10 & $0.0145(6)$ & $0.0187(6)$ & $0.0176(6)$ & $0.0031(4)$ & $0.0021(4)$ & $0.0061(4)$ \\
C23 & $0.0335(8)$ & $0.0297(7)$ & $0.0245(7)$ & $-0.0006(6)$ & $0.0050(6)$ & $0.0109(6)$ \\
C15 & $0.0181(6)$ & $0.0186(6)$ & $0.0164(6)$ & $0.0026(5)$ & $-0.0014(5)$ & $0.0026(4)$ \\
C1 & $0.0181(6)$ & $0.0167(6)$ & $0.0203(6)$ & $0.0012(5)$ & $-0.0015(5)$ & $0.0037(4)$ \\
C24 & $0.0296(7)$ & $0.0235(7)$ & $0.0233(7)$ & $0.0028(6)$ & $-0.0010(6)$ & $0.0018(5)$ \\
& & & & & \\
\hline
\end{tabular}

Geometric parameters $\left(\AA,{ }^{\circ}\right)$

\begin{tabular}{|c|c|c|c|}
\hline $\mathrm{O} 3-\mathrm{C} 20$ & $1.2363(15)$ & $\mathrm{C} 2-\mathrm{C} 3$ & $1.3838(16)$ \\
\hline $\mathrm{O} 1-\mathrm{C} 2$ & $1.3772(14)$ & $\mathrm{C} 22-\mathrm{C} 21$ & $1.5289(17)$ \\
\hline $\mathrm{O} 1-\mathrm{C} 1$ & $1.4341(15)$ & $\mathrm{C} 22-\mathrm{C} 23$ & $1.5213(18)$ \\
\hline $\mathrm{O} 2-\mathrm{C} 3$ & $1.3772(14)$ & $\mathrm{C} 22-\mathrm{C} 24$ & $1.5189(18)$ \\
\hline $\mathrm{O} 2-\mathrm{C} 1$ & $1.4386(15)$ & $\mathrm{C} 22-\mathrm{H} 22$ & $0.991(15)$ \\
\hline $\mathrm{N} 1-\mathrm{C} 20$ & $1.3443(16)$ & $\mathrm{C} 8-\mathrm{C} 9$ & $1.5120(16)$ \\
\hline $\mathrm{N} 1-\mathrm{C} 21$ & $1.4606(15)$ & $\mathrm{C} 8-\mathrm{H} 8$ & $1.006(15)$ \\
\hline $\mathrm{N} 1-\mathrm{H} 1$ & $0.894(16)$ & $\mathrm{C} 5-\mathrm{H} 5$ & 0.973 (16) \\
\hline $\mathrm{C} 7-\mathrm{C} 6$ & $1.4093(16)$ & $\mathrm{C} 21-\mathrm{H} 21 \mathrm{~A}$ & $0.987(16)$ \\
\hline $\mathrm{C} 7-\mathrm{C} 2$ & $1.3705(17)$ & $\mathrm{C} 21-\mathrm{H} 21 \mathrm{~B}$ & $1.004(16)$ \\
\hline $\mathrm{C} 7-\mathrm{H} 7$ & $0.984(16)$ & $\mathrm{C} 12-\mathrm{C} 13$ & $1.5269(16)$ \\
\hline $\mathrm{C} 6-\mathrm{C} 8$ & $1.5199(16)$ & $\mathrm{C} 12-\mathrm{C} 11$ & $1.5384(16)$ \\
\hline $\mathrm{C} 6-\mathrm{C} 5$ & $1.3945(16)$ & $\mathrm{C} 12-\mathrm{H} 12 \mathrm{~A}$ & $1.020(15)$ \\
\hline $\mathrm{C} 14-\mathrm{C} 13$ & $1.5300(17)$ & $\mathrm{C} 12-\mathrm{H} 12 \mathrm{~B}$ & $1.014(16)$ \\
\hline $\mathrm{C} 14-\mathrm{C} 15$ & $1.5270(17)$ & $\mathrm{C} 13-\mathrm{H} 13 \mathrm{~A}$ & 1.007 (16) \\
\hline $\mathrm{C} 14-\mathrm{H} 14 \mathrm{~A}$ & $0.988(16)$ & $\mathrm{C} 13-\mathrm{H} 13 \mathrm{~B}$ & 1.003 (17) \\
\hline C14-H14B & $1.021(16)$ & $\mathrm{C} 9-\mathrm{C} 10$ & $1.3250(17)$ \\
\hline $\mathrm{C} 18-\mathrm{C} 19$ & $1.3216(18)$ & C9- & $0.973(15)$ \\
\hline $\mathrm{C} 18-\mathrm{C} 17$ & $1.5007(16)$ & $\mathrm{C} 11-\mathrm{C} 10$ & $1.5024(16)$ \\
\hline C18-H18 & $0.994(15)$ & $\mathrm{C} 11-\mathrm{H} 11$ & $1.006(15)$ \\
\hline $\mathrm{C} 19-\mathrm{C} 20$ & $1.4944(16)$ & $\mathrm{C} 10-\mathrm{H} 10$ & $0.988(16)$ \\
\hline C19-H19 & $0.970(17)$ & $\mathrm{C} 23-\mathrm{H} 23 \mathrm{~A}$ & $0.98(2)$ \\
\hline $\mathrm{C} 4-\mathrm{C} 3$ & $1.3732(17)$ & $\mathrm{C} 23-\mathrm{H} 23 \mathrm{~B}$ & 1.025 (19) \\
\hline $\mathrm{C} 4-\mathrm{C} 5$ & $1.4050(17)$ & $\mathrm{C} 23-\mathrm{H} 23 \mathrm{C}$ & $0.99(2)$ \\
\hline $\mathrm{C} 4-\mathrm{H} 4$ & $0.978(16)$ & C15-H15A & $0.990(15)$ \\
\hline $\mathrm{C} 16-\mathrm{C} 17$ & $1.5411(16)$ & C15-H15B & $1.025(16)$ \\
\hline $\mathrm{C} 16-\mathrm{C} 11$ & $1.5421(16)$ & $\mathrm{C} 1-\mathrm{H} 1 \mathrm{~A}$ & 0.997 (16) \\
\hline $\mathrm{C} 16-\mathrm{C} 15$ & $1.5385(16)$ & $\mathrm{C} 1-\mathrm{H} 1 \mathrm{~B}$ & $0.978(16)$ \\
\hline $\mathrm{C} 16-\mathrm{H} 16$ & $1.016(15)$ & $\mathrm{C} 24-\mathrm{H} 24 \mathrm{~A}$ & $1.010(18)$ \\
\hline $\mathrm{C} 17-\mathrm{C} 8$ & $1.5640(16)$ & $\mathrm{C} 24-\mathrm{H} 24 \mathrm{~B}$ & $0.980(18)$ \\
\hline C17-H17 & $1.002(14)$ & $\mathrm{C} 24-\mathrm{H} 24 \mathrm{C}$ & $1.010(19)$ \\
\hline
\end{tabular}




\begin{tabular}{|c|c|c|c|}
\hline $\mathrm{C} 2-\mathrm{O} 1-\mathrm{C} 1$ & $106.11(9)$ & $\mathrm{C} 4-\mathrm{C} 5-\mathrm{H} 5$ & $118.5(9)$ \\
\hline $\mathrm{C} 3-\mathrm{O} 2-\mathrm{C} 1$ & $105.76(9)$ & $\mathrm{O} 3-\mathrm{C} 20-\mathrm{N} 1$ & $123.23(11)$ \\
\hline $\mathrm{C} 20-\mathrm{N} 1-\mathrm{C} 21$ & $124.03(10)$ & $\mathrm{O} 3-\mathrm{C} 20-\mathrm{C} 19$ & $121.94(11)$ \\
\hline $\mathrm{C} 20-\mathrm{N} 1-\mathrm{H} 1$ & $119.1(10)$ & $\mathrm{N} 1-\mathrm{C} 20-\mathrm{C} 19$ & $114.83(10)$ \\
\hline $\mathrm{C} 21-\mathrm{N} 1-\mathrm{H} 1$ & $116.7(10)$ & $\mathrm{N} 1-\mathrm{C} 21-\mathrm{C} 22$ & $112.10(10)$ \\
\hline $\mathrm{C} 6-\mathrm{C} 7-\mathrm{H} 7$ & $121.2(9)$ & $\mathrm{N} 1-\mathrm{C} 21-\mathrm{H} 21 \mathrm{~A}$ & $106.5(9)$ \\
\hline $\mathrm{C} 2-\mathrm{C} 7-\mathrm{C} 6$ & $117.32(11)$ & $\mathrm{N} 1-\mathrm{C} 21-\mathrm{H} 21 \mathrm{~B}$ & $106.8(9)$ \\
\hline $\mathrm{C} 2-\mathrm{C} 7-\mathrm{H} 7$ & $121.5(9)$ & $\mathrm{C} 22-\mathrm{C} 21-\mathrm{H} 21 \mathrm{~A}$ & $112.0(9)$ \\
\hline $\mathrm{C} 7-\mathrm{C} 6-\mathrm{C} 8$ & $119.41(10)$ & $\mathrm{C} 22-\mathrm{C} 21-\mathrm{H} 21 \mathrm{~B}$ & $110.2(9)$ \\
\hline $\mathrm{C} 5-\mathrm{C} 6-\mathrm{C} 7$ & $119.69(11)$ & $\mathrm{H} 21 \mathrm{~A}-\mathrm{C} 21-\mathrm{H} 21 \mathrm{~B}$ & $109.0(12)$ \\
\hline $\mathrm{C} 5-\mathrm{C} 6-\mathrm{C} 8$ & $120.89(10)$ & $\mathrm{C} 13-\mathrm{C} 12-\mathrm{C} 11$ & $111.67(10)$ \\
\hline $\mathrm{C} 13-\mathrm{C} 14-\mathrm{H} 14 \mathrm{~A}$ & $109.7(9)$ & $\mathrm{C} 13-\mathrm{C} 12-\mathrm{H} 12 \mathrm{~A}$ & $109.2(8)$ \\
\hline $\mathrm{C} 13-\mathrm{C} 14-\mathrm{H} 14 \mathrm{~B}$ & $108.9(9)$ & $\mathrm{C} 13-\mathrm{C} 12-\mathrm{H} 12 \mathrm{~B}$ & $110.8(9)$ \\
\hline $\mathrm{C} 15-\mathrm{C} 14-\mathrm{C} 13$ & $111.11(10)$ & $\mathrm{C} 11-\mathrm{C} 12-\mathrm{H} 12 \mathrm{~A}$ & $108.9(8)$ \\
\hline $\mathrm{C} 15-\mathrm{C} 14-\mathrm{H} 14 \mathrm{~A}$ & $109.2(9)$ & $\mathrm{C} 11-\mathrm{C} 12-\mathrm{H} 12 \mathrm{~B}$ & $110.0(9)$ \\
\hline $\mathrm{C} 15-\mathrm{C} 14-\mathrm{H} 14 \mathrm{~B}$ & $109.8(9)$ & $\mathrm{H} 12 \mathrm{~A}-\mathrm{C} 12-\mathrm{H} 12 \mathrm{~B}$ & $106.0(12)$ \\
\hline $\mathrm{H} 14 \mathrm{~A}-\mathrm{C} 14-\mathrm{H} 14 \mathrm{~B}$ & $108.0(12)$ & $\mathrm{C} 14-\mathrm{C} 13-\mathrm{H} 13 \mathrm{~A}$ & $107.7(9)$ \\
\hline $\mathrm{C} 19-\mathrm{C} 18-\mathrm{C} 17$ & $128.07(11)$ & $\mathrm{C} 14-\mathrm{C} 13-\mathrm{H} 13 \mathrm{~B}$ & $109.8(9)$ \\
\hline $\mathrm{C} 19-\mathrm{C} 18-\mathrm{H} 18$ & $116.3(9)$ & $\mathrm{C} 12-\mathrm{C} 13-\mathrm{C} 14$ & $110.15(10)$ \\
\hline $\mathrm{C} 17-\mathrm{C} 18-\mathrm{H} 18$ & $115.5(9)$ & $\mathrm{C} 12-\mathrm{C} 13-\mathrm{H} 13 \mathrm{~A}$ & $110.0(9)$ \\
\hline $\mathrm{C} 18-\mathrm{C} 19-\mathrm{C} 20$ & $120.79(11)$ & $\mathrm{C} 12-\mathrm{C} 13-\mathrm{H} 13 \mathrm{~B}$ & $110.4(9)$ \\
\hline $\mathrm{C} 18-\mathrm{C} 19-\mathrm{H} 19$ & $120.9(10)$ & $\mathrm{H} 13 \mathrm{~A}-\mathrm{C} 13-\mathrm{H} 13 \mathrm{~B}$ & $108.8(12)$ \\
\hline $\mathrm{C} 20-\mathrm{C} 19-\mathrm{H} 19$ & $118.3(10)$ & $\mathrm{C} 8-\mathrm{C} 9-\mathrm{H} 9$ & $115.5(9)$ \\
\hline $\mathrm{C} 3-\mathrm{C} 4-\mathrm{C} 5$ & $116.89(11)$ & $\mathrm{C} 10-\mathrm{C} 9-\mathrm{C} 8$ & $124.58(11)$ \\
\hline $\mathrm{C} 3-\mathrm{C} 4-\mathrm{H} 4$ & $121.4(9)$ & $\mathrm{C} 10-\mathrm{C} 9-\mathrm{H} 9$ & $119.9(9)$ \\
\hline $\mathrm{C} 5-\mathrm{C} 4-\mathrm{H} 4$ & $121.7(9)$ & $\mathrm{C} 16-\mathrm{C} 11-\mathrm{H} 11$ & $107.0(8)$ \\
\hline $\mathrm{C} 17-\mathrm{C} 16-\mathrm{C} 11$ & $111.83(9)$ & $\mathrm{C} 12-\mathrm{C} 11-\mathrm{C} 16$ & $112.87(10)$ \\
\hline $\mathrm{C} 17-\mathrm{C} 16-\mathrm{H} 16$ & $106.3(8)$ & $\mathrm{C} 12-\mathrm{C} 11-\mathrm{H} 11$ & $106.4(8)$ \\
\hline $\mathrm{C} 11-\mathrm{C} 16-\mathrm{H} 16$ & $106.0(8)$ & $\mathrm{C} 10-\mathrm{C} 11-\mathrm{C} 16$ & $110.93(9)$ \\
\hline $\mathrm{C} 15-\mathrm{C} 16-\mathrm{C} 17$ & $114.84(10)$ & $\mathrm{C} 10-\mathrm{C} 11-\mathrm{C} 12$ & $110.45(10)$ \\
\hline $\mathrm{C} 15-\mathrm{C} 16-\mathrm{C} 11$ & $110.08(9)$ & $\mathrm{C} 10-\mathrm{C} 11-\mathrm{H} 11$ & $109.0(8)$ \\
\hline $\mathrm{C} 15-\mathrm{C} 16-\mathrm{H} 16$ & $107.2(8)$ & $\mathrm{C} 9-\mathrm{C} 10-\mathrm{C} 11$ & $123.82(11)$ \\
\hline $\mathrm{C} 18-\mathrm{C} 17-\mathrm{C} 16$ & $110.54(9)$ & $\mathrm{C} 9-\mathrm{C} 10-\mathrm{H} 10$ & $119.5(9)$ \\
\hline $\mathrm{C} 18-\mathrm{C} 17-\mathrm{C} 8$ & $108.26(9)$ & $\mathrm{C} 11-\mathrm{C} 10-\mathrm{H} 10$ & $116.7(9)$ \\
\hline $\mathrm{C} 18-\mathrm{C} 17-\mathrm{H} 17$ & $110.5(8)$ & $\mathrm{C} 22-\mathrm{C} 23-\mathrm{H} 23 \mathrm{~A}$ & $110.8(11)$ \\
\hline $\mathrm{C} 16-\mathrm{C} 17-\mathrm{C} 8$ & $109.30(9)$ & $\mathrm{C} 22-\mathrm{C} 23-\mathrm{H} 23 \mathrm{~B}$ & $110.0(11)$ \\
\hline $\mathrm{C} 16-\mathrm{C} 17-\mathrm{H} 17$ & $110.5(8)$ & $\mathrm{C} 22-\mathrm{C} 23-\mathrm{H} 23 \mathrm{C}$ & $110.8(11)$ \\
\hline $\mathrm{C} 8-\mathrm{C} 17-\mathrm{H} 17$ & $107.6(8)$ & $\mathrm{H} 23 \mathrm{~A}-\mathrm{C} 23-\mathrm{H} 23 \mathrm{~B}$ & $108.3(15)$ \\
\hline $\mathrm{O} 1-\mathrm{C} 2-\mathrm{C} 3$ & $109.80(10)$ & $\mathrm{H} 23 \mathrm{~A}-\mathrm{C} 23-\mathrm{H} 23 \mathrm{C}$ & $109.1(16)$ \\
\hline $\mathrm{C} 7-\mathrm{C} 2-\mathrm{O} 1$ & $127.48(10)$ & $\mathrm{H} 23 \mathrm{~B}-\mathrm{C} 23-\mathrm{H} 23 \mathrm{C}$ & $107.8(15)$ \\
\hline $\mathrm{C} 7-\mathrm{C} 2-\mathrm{C} 3$ & $122.71(11)$ & $\mathrm{C} 14-\mathrm{C} 15-\mathrm{C} 16$ & $113.29(10)$ \\
\hline $\mathrm{C} 21-\mathrm{C} 22-\mathrm{H} 22$ & $106.4(8)$ & $\mathrm{C} 14-\mathrm{C} 15-\mathrm{H} 15 \mathrm{~A}$ & $108.9(8)$ \\
\hline $\mathrm{C} 23-\mathrm{C} 22-\mathrm{C} 21$ & $110.96(11)$ & $\mathrm{C} 14-\mathrm{C} 15-\mathrm{H} 15 \mathrm{~B}$ & $110.5(9)$ \\
\hline $\mathrm{C} 23-\mathrm{C} 22-\mathrm{H} 22$ & $108.2(9)$ & $\mathrm{C} 16-\mathrm{C} 15-\mathrm{H} 15 \mathrm{~A}$ & $108.7(8)$ \\
\hline $\mathrm{C} 24-\mathrm{C} 22-\mathrm{C} 21$ & $111.12(11)$ & $\mathrm{C} 16-\mathrm{C} 15-\mathrm{H} 15 \mathrm{~B}$ & $109.8(9)$ \\
\hline $\mathrm{C} 24-\mathrm{C} 22-\mathrm{C} 23$ & $110.86(11)$ & $\mathrm{H} 15 \mathrm{~A}-\mathrm{C} 15-\mathrm{H} 15 \mathrm{~B}$ & $105.3(12)$ \\
\hline $\mathrm{C} 24-\mathrm{C} 22-\mathrm{H} 22$ & $109.2(9)$ & $\mathrm{O} 1-\mathrm{C} 1-\mathrm{O} 2$ & $108.07(9)$ \\
\hline $\mathrm{C} 6-\mathrm{C} 8-\mathrm{C} 17$ & $111.47(9)$ & $\mathrm{O} 1-\mathrm{C} 1-\mathrm{H} 1 \mathrm{~A}$ & $109.5(9)$ \\
\hline
\end{tabular}




\begin{tabular}{|c|c|c|c|}
\hline $\mathrm{C} 6-\mathrm{C} 8-\mathrm{H} 8$ & $108.3(8)$ & $\mathrm{O} 1-\mathrm{C} 1-\mathrm{H} 1 \mathrm{~B}$ & $109.9(9)$ \\
\hline $\mathrm{C} 17-\mathrm{C} 8-\mathrm{H} 8$ & $106.4(8)$ & $\mathrm{O} 2-\mathrm{C} 1-\mathrm{H} 1 \mathrm{~A}$ & $108.7(9)$ \\
\hline $\mathrm{C} 9-\mathrm{C} 8-\mathrm{C} 6$ & $111.81(9)$ & $\mathrm{O} 2-\mathrm{C} 1-\mathrm{H} 1 \mathrm{~B}$ & $108.4(9)$ \\
\hline $\mathrm{C} 9-\mathrm{C} 8-\mathrm{C} 17$ & $111.61(9)$ & $\mathrm{H} 1 \mathrm{~A}-\mathrm{C} 1-\mathrm{H} 1 \mathrm{~B}$ & $112.2(12)$ \\
\hline $\mathrm{C} 9-\mathrm{C} 8-\mathrm{H} 8$ & $107.0(9)$ & $\mathrm{C} 22-\mathrm{C} 24-\mathrm{H} 24 \mathrm{~A}$ & $110.3(10)$ \\
\hline $\mathrm{O} 2-\mathrm{C} 3-\mathrm{C} 2$ & $110.17(10)$ & $\mathrm{C} 22-\mathrm{C} 24-\mathrm{H} 24 \mathrm{~B}$ & $110.8(11)$ \\
\hline $\mathrm{C} 4-\mathrm{C} 3-\mathrm{O} 2$ & $128.50(11)$ & $\mathrm{C} 22-\mathrm{C} 24-\mathrm{H} 24 \mathrm{C}$ & $112.1(10)$ \\
\hline $\mathrm{C} 4-\mathrm{C} 3-\mathrm{C} 2$ & $121.32(11)$ & $\mathrm{H} 24 \mathrm{~A}-\mathrm{C} 24-\mathrm{H} 24 \mathrm{~B}$ & $106.4(14)$ \\
\hline $\mathrm{C} 6-\mathrm{C} 5-\mathrm{C} 4$ & $122.06(11)$ & $\mathrm{H} 24 \mathrm{~A}-\mathrm{C} 24-\mathrm{H} 24 \mathrm{C}$ & $107.5(14)$ \\
\hline $\mathrm{C} 6-\mathrm{C} 5-\mathrm{H} 5$ & $119.5(9)$ & $\mathrm{H} 24 \mathrm{~B}-\mathrm{C} 24-\mathrm{H} 24 \mathrm{C}$ & $109.5(14)$ \\
\hline $\mathrm{O} 1-\mathrm{C} 2-\mathrm{C} 3-\mathrm{O} 2$ & $0.14(14)$ & $\mathrm{C} 3-\mathrm{O} 2-\mathrm{C} 1-\mathrm{O} 1$ & $2.82(12)$ \\
\hline $\mathrm{O} 1-\mathrm{C} 2-\mathrm{C} 3-\mathrm{C} 4$ & $178.82(11)$ & $\mathrm{C} 3-\mathrm{C} 4-\mathrm{C} 5-\mathrm{C} 6$ & $0.73(18)$ \\
\hline $\mathrm{C} 7-\mathrm{C} 6-\mathrm{C} 8-\mathrm{C} 17$ & $89.64(12)$ & $\mathrm{C} 5-\mathrm{C} 6-\mathrm{C} 8-\mathrm{C} 17$ & $-88.97(13)$ \\
\hline $\mathrm{C} 7-\mathrm{C} 6-\mathrm{C} 8-\mathrm{C} 9$ & $-36.07(14)$ & $\mathrm{C} 5-\mathrm{C} 6-\mathrm{C} 8-\mathrm{C} 9$ & $145.33(11)$ \\
\hline $\mathrm{C} 7-\mathrm{C} 6-\mathrm{C} 5-\mathrm{C} 4$ & $-0.73(18)$ & $\mathrm{C} 5-\mathrm{C} 4-\mathrm{C} 3-\mathrm{O} 2$ & $178.46(11)$ \\
\hline $\mathrm{C} 7-\mathrm{C} 2-\mathrm{C} 3-\mathrm{O} 2$ & $-179.54(11)$ & $\mathrm{C} 5-\mathrm{C} 4-\mathrm{C} 3-\mathrm{C} 2$ & $0.05(18)$ \\
\hline $\mathrm{C} 7-\mathrm{C} 2-\mathrm{C} 3-\mathrm{C} 4$ & $-0.87(19)$ & $\mathrm{C} 20-\mathrm{N} 1-\mathrm{C} 21-\mathrm{C} 22$ & $103.43(13)$ \\
\hline $\mathrm{C} 6-\mathrm{C} 7-\mathrm{C} 2-\mathrm{O} 1$ & $-178.77(11)$ & $\mathrm{C} 21-\mathrm{N} 1-\mathrm{C} 20-\mathrm{O} 3$ & $2.70(18)$ \\
\hline $\mathrm{C} 6-\mathrm{C} 7-\mathrm{C} 2-\mathrm{C} 3$ & $0.86(17)$ & $\mathrm{C} 21-\mathrm{N} 1-\mathrm{C} 20-\mathrm{C} 19$ & $-178.39(10)$ \\
\hline $\mathrm{C} 6-\mathrm{C} 8-\mathrm{C} 9-\mathrm{C} 10$ & $140.17(12)$ & $\mathrm{C} 12-\mathrm{C} 11-\mathrm{C} 10-\mathrm{C} 9$ & $-109.46(13)$ \\
\hline $\mathrm{C} 18-\mathrm{C} 19-\mathrm{C} 20-\mathrm{O} 3$ & $-5.24(18)$ & $\mathrm{C} 13-\mathrm{C} 14-\mathrm{C} 15-\mathrm{C} 16$ & $-55.96(13)$ \\
\hline $\mathrm{C} 18-\mathrm{C} 19-\mathrm{C} 20-\mathrm{N} 1$ & $175.83(11)$ & $\mathrm{C} 13-\mathrm{C} 12-\mathrm{C} 11-\mathrm{C} 16$ & $54.57(13)$ \\
\hline $\mathrm{C} 18-\mathrm{C} 17-\mathrm{C} 8-\mathrm{C} 6$ & $70.41(12)$ & $\mathrm{C} 13-\mathrm{C} 12-\mathrm{C} 11-\mathrm{C} 10$ & $179.40(10)$ \\
\hline $\mathrm{C} 18-\mathrm{C} 17-\mathrm{C} 8-\mathrm{C} 9$ & $-163.77(10)$ & $\mathrm{C} 11-\mathrm{C} 16-\mathrm{C} 17-\mathrm{C} 18$ & $-179.76(9)$ \\
\hline $\mathrm{C} 19-\mathrm{C} 18-\mathrm{C} 17-\mathrm{C} 16$ & $135.66(13)$ & $\mathrm{C} 11-\mathrm{C} 16-\mathrm{C} 17-\mathrm{C} 8$ & $61.18(12)$ \\
\hline $\mathrm{C} 19-\mathrm{C} 18-\mathrm{C} 17-\mathrm{C} 8$ & $-104.64(14)$ & $\mathrm{C} 11-\mathrm{C} 16-\mathrm{C} 15-\mathrm{C} 14$ & $52.15(13)$ \\
\hline $\mathrm{C} 16-\mathrm{C} 17-\mathrm{C} 8-\mathrm{C} 6$ & $-169.12(9)$ & $\mathrm{C} 11-\mathrm{C} 12-\mathrm{C} 13-\mathrm{C} 14$ & $-56.40(13)$ \\
\hline $\mathrm{C} 16-\mathrm{C} 17-\mathrm{C} 8-\mathrm{C} 9$ & $-43.30(12)$ & $\mathrm{C} 23-\mathrm{C} 22-\mathrm{C} 21-\mathrm{N} 1$ & $165.79(11)$ \\
\hline $\mathrm{C} 16-\mathrm{C} 11-\mathrm{C} 10-\mathrm{C} 9$ & $16.46(16)$ & $\mathrm{C} 15-\mathrm{C} 14-\mathrm{C} 13-\mathrm{C} 12$ & $56.89(13)$ \\
\hline $\mathrm{C} 17-\mathrm{C} 18-\mathrm{C} 19-\mathrm{C} 20$ & $173.97(11)$ & $\mathrm{C} 15-\mathrm{C} 16-\mathrm{C} 17-\mathrm{C} 18$ & $-53.39(13)$ \\
\hline $\mathrm{C} 17-\mathrm{C} 16-\mathrm{C} 11-\mathrm{C} 12$ & $77.81(12)$ & $\mathrm{C} 15-\mathrm{C} 16-\mathrm{C} 17-\mathrm{C} 8$ & $-172.45(9)$ \\
\hline $\mathrm{C} 17-\mathrm{C} 16-\mathrm{C} 11-\mathrm{C} 10$ & $-46.76(13)$ & $\mathrm{C} 15-\mathrm{C} 16-\mathrm{C} 11-\mathrm{C} 12$ & $-51.11(13)$ \\
\hline $\mathrm{C} 17-\mathrm{C} 16-\mathrm{C} 15-\mathrm{C} 14$ & $-75.12(13)$ & $\mathrm{C} 15-\mathrm{C} 16-\mathrm{C} 11-\mathrm{C} 10$ & $-175.68(10)$ \\
\hline $\mathrm{C} 17-\mathrm{C} 8-\mathrm{C} 9-\mathrm{C} 10$ & $14.53(16)$ & $\mathrm{C} 1-\mathrm{O} 1-\mathrm{C} 2-\mathrm{C} 7$ & $-178.70(12)$ \\
\hline $\mathrm{C} 2-\mathrm{O} 1-\mathrm{C} 1-\mathrm{O} 2$ & $-2.74(12)$ & $\mathrm{C} 1-\mathrm{O} 1-\mathrm{C} 2-\mathrm{C} 3$ & $1.63(13)$ \\
\hline $\mathrm{C} 2-\mathrm{C} 7-\mathrm{C} 6-\mathrm{C} 8$ & $-178.69(10)$ & $\mathrm{C} 1-\mathrm{O} 2-\mathrm{C} 3-\mathrm{C} 4$ & $179.60(12)$ \\
\hline $\mathrm{C} 2-\mathrm{C} 7-\mathrm{C} 6-\mathrm{C} 5$ & $-0.07(17)$ & $\mathrm{C} 1-\mathrm{O} 2-\mathrm{C} 3-\mathrm{C} 2$ & $-1.84(13)$ \\
\hline $\mathrm{C} 8-\mathrm{C} 6-\mathrm{C} 5-\mathrm{C} 4$ & $177.87(11)$ & $\mathrm{C} 24-\mathrm{C} 22-\mathrm{C} 21-\mathrm{N} 1$ & $-70.39(13)$ \\
\hline $\mathrm{C} 8-\mathrm{C} 9-\mathrm{C} 10-\mathrm{C} 11$ & $-0.56(19)$ & & \\
\hline
\end{tabular}

Hydrogen-bond geometry $\left(\AA,{ }^{\circ}\right)$

$\mathrm{Cg} 2$ is the centroid of the benzene ring $\mathrm{C} 2-\mathrm{C} 7$.

\begin{tabular}{lllll}
\hline$D-\mathrm{H}^{\cdots} A$ & $D-\mathrm{H}$ & $\mathrm{H}^{\cdots} A$ & $D \cdots A$ & $D-\mathrm{H}^{\cdots} A$ \\
\hline $\mathrm{N} 1-\mathrm{H} 1 \cdots \mathrm{O} 3^{\mathrm{i}}$ & $0.896(16)$ & $2.105(16)$ & $2.8938(13)$ & $146.3(13)$
\end{tabular}


supporting information

$\mathrm{C} 7-\mathrm{H} 7 \cdots \mathrm{O} 1^{\mathrm{ii}}$

$\mathrm{C} 1-\mathrm{H} 1 B \cdots C g 2^{\mathrm{i}}$

Symmetry codes: (i) $x-1, y, z$; (ii) $-x+1,-y+2,-z+1$.
$0.984(16)$

$0.978(16)$
$2.503(16)$

$2.595(15)$
$3.4264(15)$

$3.4578(12)$
$156.3(12)$

$147.4(11)$ 